\title{
Kinetics of the boron-oxygen related defect in theory and experiment
}

\author{
Axel Herguth ${ }^{\text {a) }}$ and Giso Hahn \\ Department of Physics, University of Konstanz, 78464 Konstanz, Germany
}

(Received 7 June 2010; accepted 21 October 2010; published online 10 December 2010)

\begin{abstract}
The formation of boron-oxygen complexes in boron-doped crystalline silicon can lead to a severe reduction in the minority charge carrier lifetime. This strongly influences, e.g., solar cell efficiencies if the material is used for photovoltaic application. Recent investigations have shown that a recovery of the carrier lifetime can be achieved by a subsequent thermally enhanced reaction induced by charge carriers. A model of the reaction dynamics of the boron-oxygen complex by means of rate equations is presented in this paper. Following a mathematical description of the reactions involved, the consequences based on the calculations are presented and allow a prediction of the observable electrical parameters. The fundamental agreement with measured data is proven experimentally for different phenomena. (C) 2010 American Institute of Physics. [doi:10.1063/1.3517155]
\end{abstract}

\section{INTRODUCTION}

The lifetime of minority charge carriers is the main indicator for the quality of crystalline silicon wafers. Besides grain boundaries which generally have a negative impact on the lifetime of multicrystalline materials, the lifetime especially of monocrystalline silicon is often limited by pointlike defects resulting from contaminations inserted either deliberately (like dopants) or unintentionally. The example of iron as contaminant demonstrates very well that even very small quantities in the order of $1-10 \times 10^{12}$ atoms $/ \mathrm{cm}^{3}$ can seriously reduce the lifetime. ${ }^{1}$ Although the purity of monocrystalline silicon produced by the Czochralski method $(\mathrm{Cz}-\mathrm{Si})$ and the floatzone method (FZ-Si) may seem comparable regarding many possible contaminants, the highest observed bulk lifetimes (at the same base doping) differ significantly in p-type material. Thus the apparent different contamination level of oxygen originating from the particular crystal pulling method comes to the fore. The concentration of (interstitial) oxygen in FZ-Si is often hardly detectable (at least by commonly used infrared absorption measurements) and thus lies in the order of $10^{16}$ atoms $/ \mathrm{cm}^{3}$ or below. However, the concentration of interstitial oxygen in $\mathrm{Cz}-\mathrm{Si}$ is generally at least one order of magnitude higher than in FZ-Si and lies in the range of $1-10 \times 10^{17}$ atoms $/ \mathrm{cm}^{3}$ and indeed oxygen is well known to be a problematic contaminant in silicon, especially in conjunction with other impurities.

\section{DYNAMICS DUE TO B-O COMPLEXES}

\section{A. Degradation}

Known since the 1970 s, ${ }^{2,3}$ boron doped Cz-Si shows an asymptotically saturating degradation of its minority charge carrier lifetime under illumination (more general if charge carriers are injected) supported by thermal energy. This effect often generally referred to as light induced degradation already sets in at room temperature even though higher temperatures enforce a faster degradation. As many investigations $^{4-7}$ in recent years have shown, the stable life-

${ }^{a)}$ Electronic mail: axel.herguth@uni-konstanz.de. time achieved after degradation scales with the boron doping as well as with the measured interstitial oxygen concentration. This finding is pointing out that at least boron and oxygen are involved in the formation of the strongly recombination active complex. The correlation of the minority charge carrier lifetime with doping and oxygen level gave rise to the empirical formula of Bothe et al. ${ }^{7}$ [Eq. (1)] enabling the prediction of the stable degraded lifetime $\tau_{d}$ in dependence of both substitutional boron $\left[\mathrm{B}_{\mathrm{s}}\right]$ and interstitial oxygen concentration $\left[\mathrm{O}_{\mathrm{i}}\right]$.

$$
\tau_{d}=7.675 \times 10^{45}\left[\mathrm{~B}_{\mathrm{S}}\right]^{-0.824}\left[\mathrm{O}_{\mathrm{i}}\right]^{-1.748}
$$

The correlation described by this equation suggests that the defect responsible for the lifetime after completed degradation consists of one boron atom and two oxygen atoms presumably bound in the form of an oxygen dimer. In contrast to single interstitial oxygen atoms, an oxygen dimer features a strongly enhanced diffusion already at low temperatures (like room temperature). This relatively fast diffusion is due to a recombination enhanced Bourgoin-Corbett diffusion mechanism, ${ }^{8}$ in which the dimer permanently changes its charge state following a geometrical reconfiguration in the silicon host lattice as different configurations for different charge states are energetically preferred. ${ }^{9}$

\section{B. The 3-state model}

Beginning in 2006, several research groups have shown that the observed degradation due to the formation of the boron-oxygen complexes is just a first step in a more complex defect formation process. ${ }^{10-14}$ Subsequent to the degradation there may occur an increase in minority charge carrier lifetime under the influence of light (or again more general if charge carriers are injected) and temperature. This so called light induced regeneration may be explained by a conversion of the strongly recombination active complex into an additional recombination inactive state of the boron-oxygen complex resulting in a more advanced 3-state model sketched in Fig. 1. Whether the complex is dissolved, undergoes a geo- 


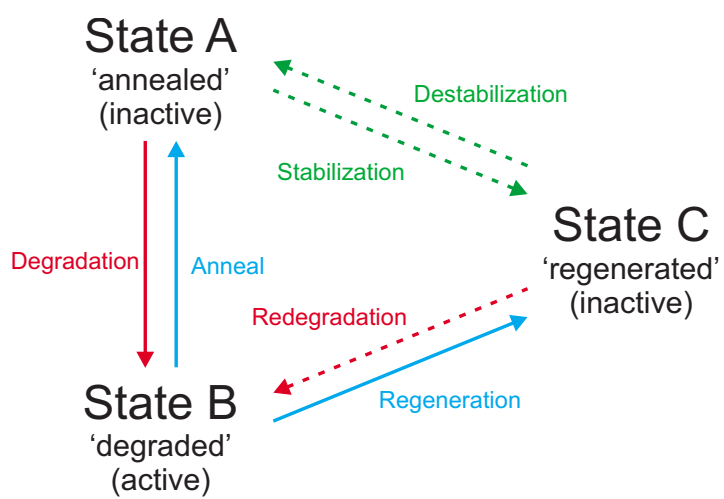

FIG. 1. (Color online) Working model of a 3-state reaction scheme describing the boron-oxygen complex (Ref. 11). Continuous arrows denote observed reactions, dashed reaction paths could not be verified so far.

metrical change or an additional species is attached to the complex is of no interest for this model in contrast to the model presented by Lim et al. ${ }^{14}$

\section{Mathematical approach}

Assuming that each reaction uses one specimen of the precursor state to form one specimen of the new state of the complex and that there is a finite number of participating complexes (concentration $n^{0}$ ) one may describe interaction of the states by a system of linear differential equations for the concentrations $n_{i}(t)$ of the different states $(i, j, \ell$ $=\mathrm{A}, \mathrm{B}, \mathrm{C}$ and $i \neq j \neq \ell$ ).

$$
\frac{\partial n_{i}}{\partial t}=-\left(\kappa_{i j}+\kappa_{i \ell}\right) n_{i}+\kappa_{j i} n_{j}+\kappa_{\ell i} n_{\ell},
$$

where $n_{i}$ is the concentration of the complexes residing in state $i$ of the 3 -state model (Fig. 1) and the $\kappa_{i j}$ are the reaction rates (in this case for the reaction from state $i$ to state $j$ ). Reactions with the precursor state equaling the successor state $(i=j)$ do not change the system and therefore are excluded $\left(\kappa_{i i}=0\right.$ for $\left.i=\mathrm{A}, \mathrm{B}, \mathrm{C}\right)$. The complete time dependent solution of the concentrations of the different states of the complex reads as follows:

$$
n_{i}(t)=n_{i}^{\infty}+n_{i}^{+} e^{-(\Omega-\omega) t}-n_{i}^{-} e^{-(\Omega+\omega) t} .
$$

The effective reaction rates $(\Omega-\omega)$ and $(\Omega+\omega)$ of the exponential functions are given by a combination of the reaction rates $\kappa_{i j}($ with $i \neq j)$.

$$
\begin{aligned}
& \Omega=\frac{1}{2} \sum_{\substack{i, j=\mathrm{A}, \mathrm{B}, \mathrm{C} \\
i \neq j}} \kappa_{i j} \\
& \text { and } \omega^{2}=\Omega^{2}-\sum_{\substack{i, j, \ell=\mathrm{A}, \mathrm{B}, \mathrm{C} \\
i \neq j \neq \ell}}\left(\kappa_{i j} \kappa_{j \ell}+\frac{1}{2} \kappa_{i j} \kappa_{\ell j}\right) .
\end{aligned}
$$

The concentration coefficients $n_{i}^{\infty}$ and $n_{i}^{ \pm}$(with $i=\mathrm{A}, \mathrm{B}, \mathrm{C}$ ) are determined by the reaction rates $\kappa_{i j}$ (with $i \neq j$ ) as well as the starting conditions $n_{i}^{0}=n_{i}(t=0)$ and the total concentration $n^{0}$ participating in the reactions.

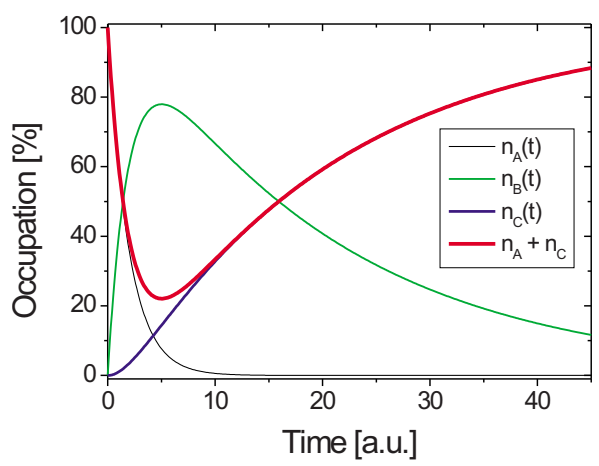

FIG. 2. (Color online) Calculated temporal progression of the relative occupation of the different states of the complex using the derived formula of the 3 -state model with $\kappa_{\mathrm{AB}}=10 \cdot \kappa_{\mathrm{BC}}$ and vanishing other reaction rates. Due to a similarly low recombination activity, a mixture of the annealed state $\mathrm{A}$ and the regenerated state $\mathrm{C}$ might not be distinguishable and thus only the envelope $n_{\mathrm{A}}+n_{\mathrm{C}}$ will be observable.

$$
\begin{aligned}
n_{i}^{\infty}= & \frac{n^{0}}{\Omega^{2}+\omega^{2}} \cdot\left(\kappa_{j i} \kappa_{\ell i}+\kappa_{j i} \kappa_{\ell j}+\kappa_{\ell i} \kappa_{j \ell}\right), \\
n_{i}^{ \pm}= & \frac{1}{2}\left(\frac{\Omega}{\omega} \pm 1\right)\left(n_{i}^{0}-n_{i}^{\infty}\right) \\
& +\frac{1}{2 \omega}\left[-\left(\kappa_{i j}+\kappa_{i \ell}\right) n_{i}^{0}+\kappa_{j i} n_{j}^{0}+\kappa_{\ell i} n_{\ell}^{0}\right],
\end{aligned}
$$

where $n_{i}^{\infty}$ is obviously the asymptotic limiting value of the concentration for longer times approaching infinity when the exponential functions vanish. The equation has the shape of two superimposed exponential functions. Figure 2 shows a principle progression of the occupation [ratio $n_{i}(t)$ to $\left.n^{0}\right]$ of the three states and also of the envelope of the states A and $\mathrm{C}$ which corresponds to the measured values as these states exhibit similarly low recombination activities. As these equations in general do not represent the measured values in the experiments, the time dependent solution for the occupation of the different states has to be converted to measurable values like the minority charge carrier lifetime. The effective lifetime may be expressed by a sum of different factors such as surface recombination $\tau_{\text {sur }}$ and different recombination channels in the bulk denoted here as $\tau_{b g}$ for any background limitations as well as the lifetimes related to the defect states $\mathrm{A}, \mathrm{B}$, and $\mathrm{C}$.

$$
\frac{1}{\tau_{\text {eff }}}=\frac{1}{\tau_{\text {sur }}}+\frac{1}{\tau_{\mathrm{A}}}+\frac{1}{\tau_{\mathrm{B}}}+\frac{1}{\tau_{\mathrm{C}}}+\frac{1}{\tau_{b g}} .
$$

In the case of standard $\mathrm{Cz}$ material, the recombination active state B plays the important role and, as experiments suggest, ${ }^{10,11}$ the defect states $\mathrm{A}$ and $\mathrm{C}$ seem to be that inactive that their correlated lifetimes $\tau_{\mathrm{A}}$ and $\tau_{\mathrm{C}}$ are larger than the background $\tau_{b g}$ and can be neglected compared to the term $\tau_{b g}$. However, a constant effective background lifetime $\tau_{b g}^{*}$ may be chosen to take this into account and to get rid of the surface influence in Eq. (7) as well. According to the defect driven Shockley-Read-Hall recombination mechanism, ${ }^{15}$ the lifetime $\tau_{\mathrm{B}}$ correlated with the degraded state B depends inversely on the concentration of defects $n_{\mathrm{B}}: \tau_{\mathrm{B}} \propto 1 / n_{\mathrm{B}}$ and thus using the expression $n_{\mathrm{B}}(t)$ from Eq. (3) (with $i=\mathrm{B}$ ) the 


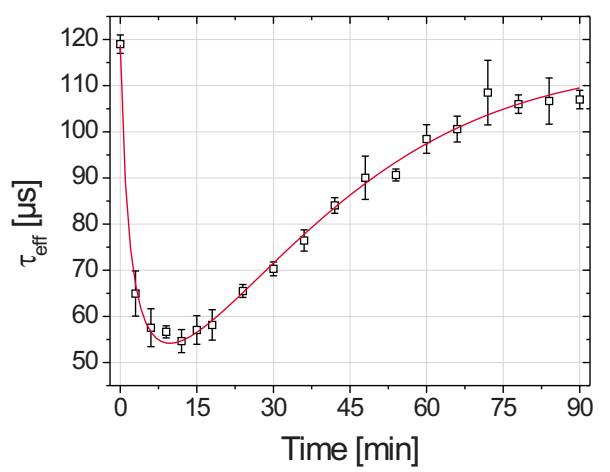

FIG. 3. (Color online) Lifetime data measured by QSSPC at the same excess carrier density $\left(1.2 \times 10^{15} \mathrm{~cm}^{-3}\right)$, the line represents a fit according to the 3 -state model to the data.

time dependent effective measurable lifetime may be expressed as follows:

$$
\tau_{\mathrm{eff}}(t)=\left[\frac{1}{\tau_{b g}^{*}}+\frac{1}{\tau^{+}} e^{-(\Omega-\omega) t}-\frac{1}{\tau^{-}} e^{-(\Omega+\omega) t}\right]^{-1} .
$$

Equation (8) was applied on lifetime data measured by the quasi-steady-state photoconductance (QSSPC) method $^{16}$ taken at one and the same excess carrier density and the result is shown in Fig. 3. The theoretical curve matches the measured data very well especially taking the measurement uncertainty into account. Note that the specific reaction rates $(\Omega-\omega)$ and $(\Omega+\omega)$ themselves do not represent the pure time constants of degradation and regeneration even if the exponential terms are responsible for the different parts of the superimposed progression. How complicated the extraction of the pure time constants really is may be demonstrated in a single example. Assuming that the reactions of direct stabilization $(A \Rightarrow C$ in Fig. 1), redegradation $(C \Rightarrow B)$ and destabilization $(\mathrm{C} \Rightarrow \mathrm{A})$ play no role in an experiment, their reaction constants vanish $\left(\kappa_{\mathrm{CB}}=\kappa_{\mathrm{CA}}=\kappa_{\mathrm{AC}}=0\right)$. Even then it can be calculated [Eq. (4)] that the observed reaction rates $(\Omega-\omega)$ and $(\Omega+\omega)$ are still dependent on the remaining three reaction rates $\kappa_{i j}$ in the form

$$
\Omega=\frac{1}{2}\left(\kappa_{\mathrm{AB}}+\kappa_{\mathrm{BA}}+\kappa_{\mathrm{BC}}\right) \quad \text { and } \omega=\sqrt{\Omega^{2}-\kappa_{\mathrm{AB}} \kappa_{\mathrm{BC}}} .
$$

Only for a negligible anneal reaction $\kappa_{\mathrm{BA}}$ the reaction rates $(\Omega-\omega)$ and $(\Omega+\omega)$ match the reaction rates of degradation $\kappa_{\mathrm{AB}}$ and regeneration $\kappa_{\mathrm{BC}}$. A simulation shown in Fig. 4 shows the influence of the anneal reaction which may have a significant influence on the observable curves depending on the exact reaction constants in an experiment.

For solar cells, the electrical parameters and especially the open circuit voltage $V_{\mathrm{oc}}$ are strongly correlated with the bulk lifetime. In an idealized diode model, $V_{\mathrm{oc}}$ is determined by the short-circuit current density $j_{\mathrm{sc}}$ as well as the saturation current density $j_{0}$ of the diode and may be written as follows:

$$
V_{\mathrm{oc}}=k T / q \cdot \ln \left(j_{\mathrm{sc}} / j_{0}+1\right) .
$$

Assuming an emitter structure of high quality, $j_{0}$ is limited by recombination in the bulk and depends crucially on the minority charge carrier lifetime in the bulk. Unfortunately, $j_{\mathrm{sc}}$ depends on the lifetime as well and a generally valid analytic

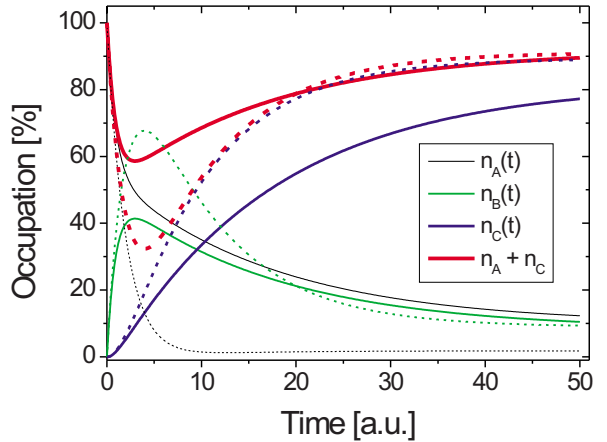

FIG. 4. (Color online) Calculated occupation of the three states during a degradation-regeneration cycle including (solid lines) and completely excluding (dashed lines) the anneal reaction. The anneal reaction may have serious influence on the progression of the degradation and regeneration cycle and may lead to incorrectly determined time constants.

expression is not derivable. Nevertheless, simulations show that an increase in the bulk lifetime leads to a gain in $V_{\mathrm{oc}}$, and even if the dependence is nonlinear, small changes in $V_{\mathrm{oc}}$ reproduce the changes in bulk lifetime. The characteristic shape of the two exponential functions in Eq. (3) remains clearly visible with small distortion. However, the determination and comparison of time constants from comparable distorted data remains valid.

\section{SAMPLE PREPARATION AND SETUP}

The experimental results presented in this paper were obtained on p-type boron doped $\mathrm{Cz}-\mathrm{Si}$ wafers with $1-2 \Omega \mathrm{cm}$ bulk resistivity and interstitial oxygen concentrations in the range of $5-10 \times 10^{17} \mathrm{~cm}^{-3}$. Both surfaces of the lifetime samples were passivated by a hydrogen-rich silicon nitride $\left(\mathrm{SiN}_{x}: \mathrm{H}\right)$ layer deposited by plasma-enhanced chemical vapor deposition (PECVD) in order to overcome the surface limitation $\left(\tau_{\text {sur }}>\tau_{\text {bulk }}\right)$. The solar cells used for $V_{\text {oc }}$ measurements were processed according to a standard industrialtype fabrication scheme. After saw damage etching a $\mathrm{POCl}_{3}$ emitter (sheet resistivity around $50 \Omega / \mathrm{sq}$ ) was formed on both sides of the standard $125 \times 125 \mathrm{~mm}^{2}$ semisquare wafer format. A $75 \mathrm{~nm}$ thick hydrogen-rich $\mathrm{SiN}_{x}: \mathrm{H}$ layer (refractive index at $600 \mathrm{~nm}$ around 2.0) was deposited by PECVD on the front side. Metallization was realized by screen printing of metal pastes (Ag grid on the front side and $\mathrm{Al}$ on the rear). A cofiring step in a belt furnace served for contact formation. Edge isolation was carried out by a wafer dicing saw.

Lifetime measurements were taken with a WT-100 QSSPC system at room temperature. $V_{\text {oc }}$ was measured under constant illumination and standard test conditions $\left(1 \mathrm{~kW} / \mathrm{m}^{2}\right.$, approximately AM $1.5 \mathrm{G}$ spectrum, $\left.{ }^{17} 2{ }^{\circ} \mathrm{C}\right)$. Temperature steps applied to the samples between the measurements were carried out on a stabilized hot plate $(\Delta T$ $<1 \mathrm{~K}$ ). Halogen lamps (as black body like thermal light sources) were used for illumination during temperature steps, intensity variation was within $5 \%_{\text {rel }}$. External currents (without illumination) were regulated with a deviation down to $1 \%$. 


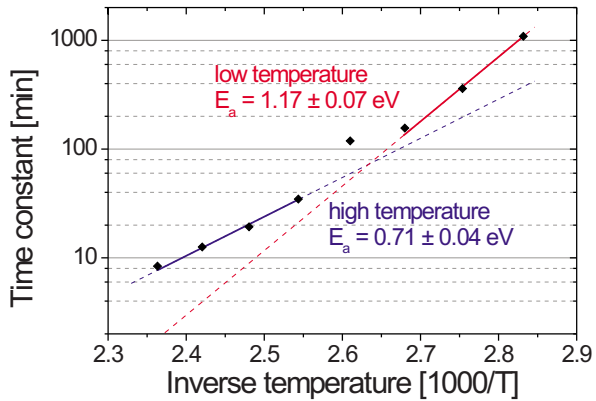

FIG. 5. (Color online) Arrhenius plot in logarithmic scaling for the determination of the activation energy for the regeneration reaction. Fitting the whole temperature range with one activation energy is problematic. Even different fits for different temperature ranges might be questionable.

\section{DEPENDENCES OF THE REACTION RATES}

\section{A. Regeneration and temperature}

To describe the observed reactions involving the regenerated state $\mathrm{C}$, a specific time constant (inverse reaction rate) may be extracted from the measured lifetime data as described in the previous section. It is found that charge carrier injection and temperature are two easily accessible parameters to alter the time constant of the regeneration reaction. A more detailed investigation of this time constant on the temperature dependence is presented in Fig. 5 showing the strong influence of temperature on the time constant. As many reactions with a strong temperature dependence, it might be assumed that the inverse time constant $\kappa$ (or better called reaction rate) follows the Arrhenius law in the form

$$
\kappa=\nu_{0} \cdot \exp \left(-E_{a} / k T\right)
$$

giving a specific thermal activation energy $E_{a}$ and some type of trial frequency $\nu_{0}$ for the conversion of a precursor state into the successor state. As Fig. 5 shows, this was tested in an experiment over a broad temperature range.

The interesting point is that a fit with the Arrhenius formula is not that simple as it seems. Depending on the temperature range the fits result in notably different values for the activation energy. Two possible explanations may be given for this. First there might be some experimental error which in principle could not be ruled out completely. The other explanation queries the validity of the Arrhenius law in this special case. The Arrhenius law focuses on thermally activated reactions and the exponential function originates from the thermal Boltzmann statistic. As the reaction seems to be more complicated, this might be a too simple approach and even if the exponential term is correct in principle, there could be also a temperature dependence of the trial frequency itself rendering the use of the Arrhenius law in Fig. 5 questionable.

\section{B. Regeneration and carrier injection}

As implied further, there is also a dependence of the time constant of regeneration on the light intensity or more precisely on excess charge carrier density. An investigation of both light intensity as well as biased current (densities) is shown in Fig. 6.
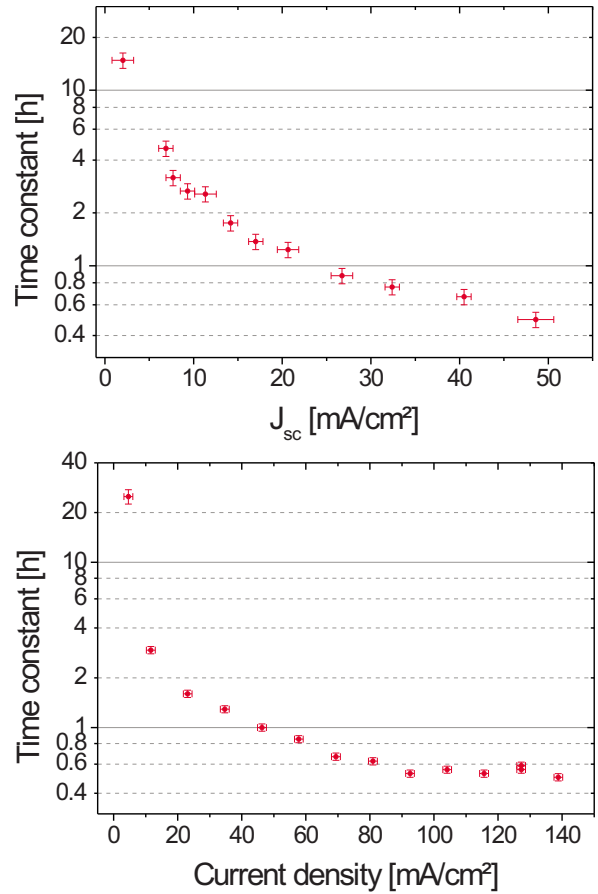

FIG. 6. (Color online) Time constant of the regeneration reaction determined for excess carriers generated by light (top) and external bias currents (bottom) at $120{ }^{\circ} \mathrm{C}$. In principle, both data sets point toward a saturation at higher injection levels. A direct comparison is difficult due to a different distribution of charge carriers for the two injection methods.

As can be seen, in principle a higher excess carrier density leads to a faster regeneration process and in contrast to the degradation process which seems to be influenced only by light intensity below $1 / 100$ sun, ${ }^{18}$ the time constant of regeneration still shows a significant change at illumination intensities around 1 sun. However, a further discussion and comparison of the different carrier injection modes is more complicated as light excitation and external biasing result in a different charge carrier distribution within the cell which could be of inherent importance. The main question still to be solved is what influence charge carriers may have on the conversion of the defect.

\section{Destabilizing the regenerated state}

Whatever happened to the boron-oxygen complex during the regeneration process might also be reversed or may be subject to further alteration. Exactly this could be proven at temperatures around $200{ }^{\circ} \mathrm{C}$ if the regeneration process is disabled due to the lack of charge carrier excitation. The regenerated state becomes instable and converts into a form which again shows the degradation behavior as shown in Fig. 7.

Therefore, it is assumed that destabilizing the regenerated state $\mathrm{C}$ leads perhaps with an intermediate stage to the annealed state A of the reaction scheme in Fig. 1. Again this reaction was evaluated using the Arrhenius law to determine a specific activation energy $E_{a}$ which is shown in Fig. 8. 


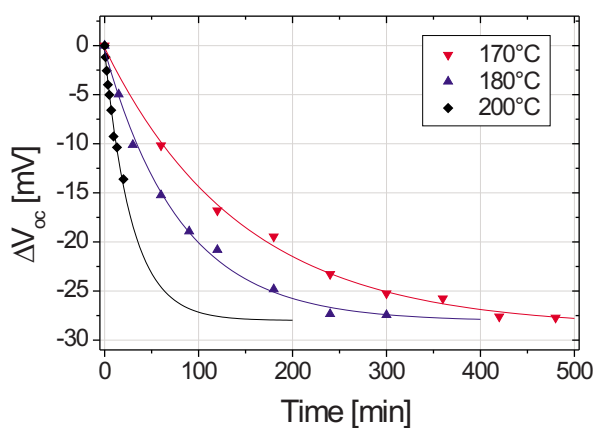

FIG. 7. (Color online) The destabilization reaction at elevated temperatures made visible by a subsequent degradation experiment on standard industrialtype solar cells. A drop in open circuit voltage $V_{\text {oc }}$ shows the increasing destabilization for different temperatures. The lines are guides to the eye.

\section{CONSEQUENCES AND PREDICTIONS}

\section{A. Agreement with the model}

As the main parameters of most reactions within the 3 -state model are known, the model can be used to predict some properties not discussed so far. As a first consequence, the "amplitude" or the maximal drop of the minority charge carrier lifetime or the solar cell's electrical parameters is determined by the ratio of the reaction constants of the degradation and regeneration processes. If the regeneration process is very slow compared to the degradation process (using a low temperature and low injection) then the degradation will almost completely take place before regeneration starts and thus the highest amplitude may be observed. As can be seen from the simulations in Fig. 9 the observable amplitude (which is the occupation of state A and C together) is significantly reduced if the ratio of the reaction constants of regeneration and degradation increases meaning that the boronoxygen complexes residing in the degraded state $\mathrm{B}$ are converted that fast into the regenerated state $\mathrm{C}$ that they cannot accumulate in large numbers.

In reality the observed time constants of the regeneration process are smaller than for the degradation process and the observable temporal progression of the electrical parameters of the samples should comply more with the larger amplitude curve (red) in Fig. 9. This effect is measurable using different excess carrier density levels exceeding 1/100 sun, where

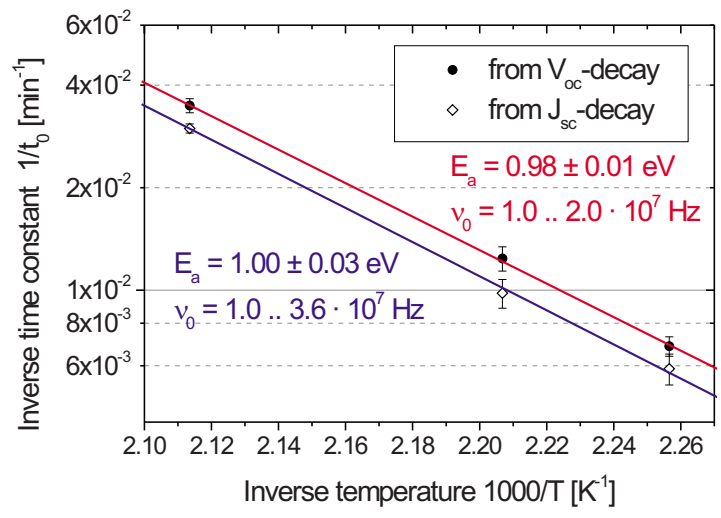

FIG. 8. (Color online) Arrhenius plot using the time constants $t_{0}$ derived from exponential decay fits on the data shown in Fig. 7 giving the activation energy of the destabilization process.

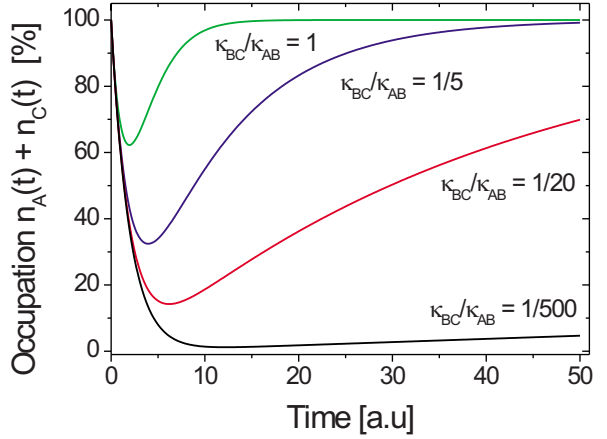

FIG. 9. (Color online) Calculated curves for the degradation-regeneration cycle using different ratios of the correlated reaction constants. A relatively fast regeneration process leads to a reduction in the observable amplitude.

the light intensity dependence of the degradation process saturates, ${ }^{18}$ and a constant elevated temperature. Figure 10 shows a lifetime measurement series demonstrating this effect even if in this case different temperatures were used. The degradation speeds up also due to different temperatures but the ratio of the time constants of regeneration and degradation changes.

\section{B. Mixed state equilibriums}

As a further consequence of a continuous conversion of all states into each other (even on indirect paths) it can be assumed that there exist also mixed state equilibriums, that means that the macroscopic sample exhibits the boronoxygen complex in different states at the same time but the reactions are balanced so far, that no temporal change occurs any more. The development of this equilibrium can be simulated and is shown in Fig. 11. Obviously the ratio of regeneration and destabilization reaction rates determines the final occupation of the inactive states $\mathrm{A}$ and $\mathrm{C}$ and thus also the occupation of the lifetime limiting state B.

If the destabilization process dominates over the regeneration process which should occur at low excess carrier densities suppressing the regeneration compared to the degradation process (the influence of light intensity saturates around $1 / 100$ sun for the degradation process ${ }^{18}$ ), the equilibrium shifts toward the degraded state $\mathrm{B}$. If the regeneration process dominates over the destabilization process which means high injection levels at relatively low temperatures, the equi-

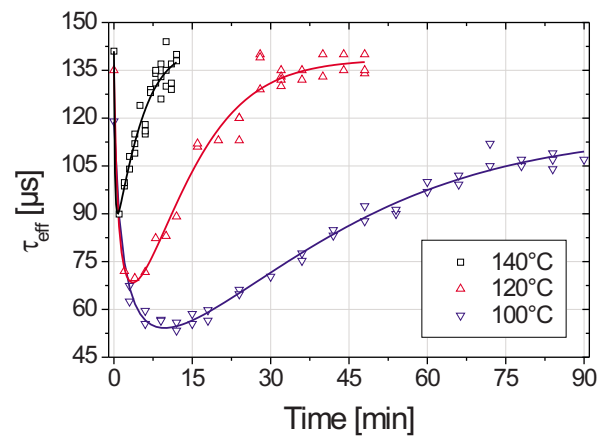

FIG. 10. (Color online) Lifetime data measured by QSSPC showing the degradation-regeneration cycle at different temperatures. As the ratio of the reaction constants changes, a loss in the observed amplitude becomes visible for higher temperatures. 


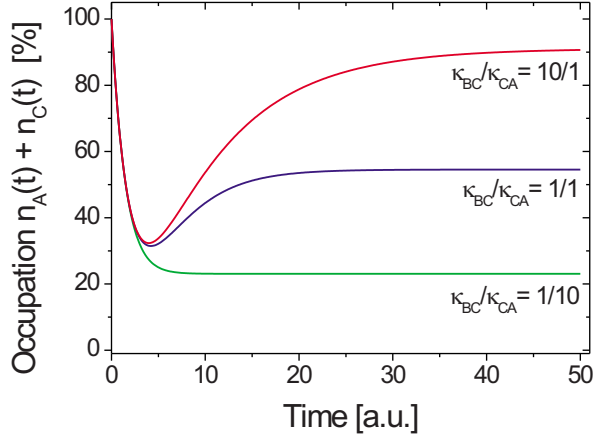

FIG. 11. (Color online) Calculated curves for different ratios of the reaction constants of the regeneration and destabilization process. A relatively strong destabilization leads to a macroscopically mixed state identifiable by an asymptotic value significantly below the starting level.

librium shifts toward the regenerated state C. For all conditions between the two extreme cases, a macroscopically mixed state should occur. Figure 12 shows a simulation using the Arrhenius type reaction rates at a fixed external excitation of one sun and a measurement series showing the stable (equilibrium) electrical parameters of a solar cell after regeneration treatment at different temperatures.

An experiment based on the simulations was carried out. The regeneration treatment took place at different temperatures in the range of $160-230{ }^{\circ} \mathrm{C}$ under illumination of 1 sun. The partly existent annealed state of the complex was degraded at low temperature after the regeneration treatment to distinguish between the stable regenerated state $\mathrm{C}$ and the instable (degradable) annealed state A. The results are shown in Fig. 13. Even if the experiment could not be performed at temperatures exceeding $230{ }^{\circ} \mathrm{C}$, a significant increase in $V_{\mathrm{oc}}$ in the subsequent degradation process indicates that the equilibrium does not longer prefer the regenerated state $\mathrm{C}$ [as shown in Fig. 12 for the $n_{\mathrm{C}}(T)$ curve]. Note at this point that the time constant of the regeneration process can be influenced by more external parameters than temperature alone (e.g., Fig. 6) and thus the experiment is in equilibrium only for these very specific experimental conditions. It is clear that altering the time constant of the regeneration process will shift the cross-over point of the occupation of state $\mathrm{C}$ and state A in Fig. 12 toward higher or lower temperatures.

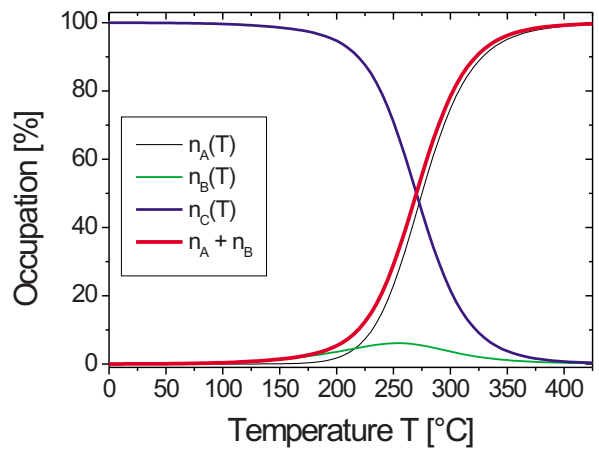

FIG. 12. (Color online) Calculated values of the occupation of the three states vs temperature using the reaction constants obtained from above experiments. For temperatures exceeding $\sim 150{ }^{\circ} \mathrm{C}$, the macroscopic system prefers a mixed state and higher temperatures lead to a completely not regenerated mixed state.

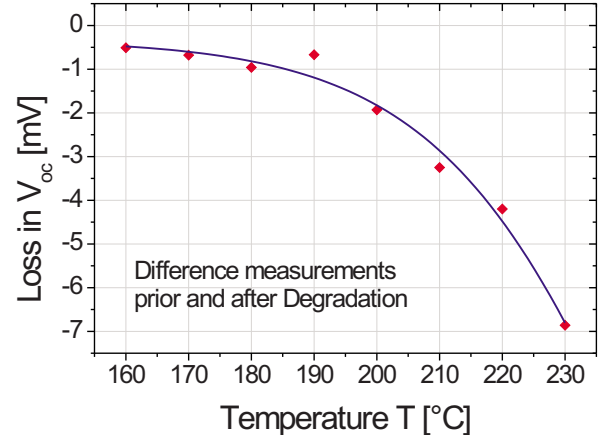

FIG. 13. (Color online) Mixed states made visible experimentally on solar cell level by a complete regeneration process and subsequent degradation to distinguish between the instable annealed state $\mathrm{A}$ and the stable regenerated state $\mathrm{C}$. The increasing drop in $V_{\mathrm{oc}}$ with temperature is a sign of this phenomenon as predicted in Fig. 12.

\section{The path of destabilization}

One more conclusion drawn from the 3-state model pertains the destabilization reaction which is induced by a heat treatment without any excess carriers. From the reaction scheme there appear to be two possible ways for a boronoxygen complex to convert from the regenerated state $\mathrm{C}$ to the annealed state $\mathrm{A}$. The easiest way would be that the state converts directly via the destabilization path. A more complex path would be a double reaction with state B as intermediate stage on the way from state $\mathrm{C}$ to state A. Alternatively, another path exceeding the model could be imaginable but is not further traced here. Based on the equations of the 3-state model [Eqs. (3)-(6)] a principally simulation (Fig. 14) was carried out to analyze the occupation of states for the first two cases.

As can be seen, both possible paths lead to the expected reduction in the occupation of state $\mathrm{C}$ but more important at this point is the occupation of state A and state B. For the direct transition from state $\mathrm{C}$ to state $\mathrm{A}$ (solid curves) the occupation of state B remains zero all the time as expected. In contrast the double reaction (dashed curves) leads to a finite occupation of the degraded state B for a certain period of time. Thus this could offer a possibility to clarify the exact conversion path leading from the regenerated state $\mathrm{C}$ to the annealed state A. As all reactions are temperature dependent, it is clear that observable curves of the described reaction

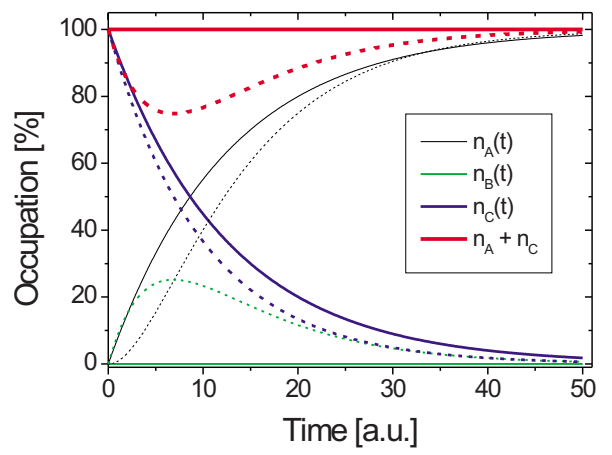

FIG. 14. (Color online) Calculated time dependent occupation of the three states for a direct destabilization (solid lines) and a double reaction using the degraded state as intermediate stage (dashed lines). The significant difference is the finite occupation of state B in the second case. 


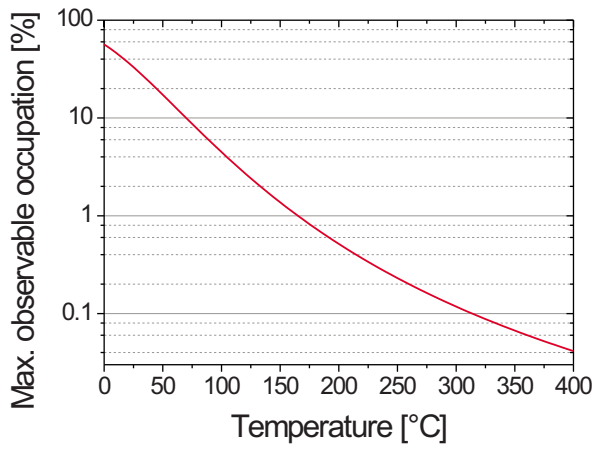

FIG. 15. (Color online) Calculated maximum observable amplitude of state B occupation vs temperature using Arrhenius type equations for extrapolation. Lower temperatures lead to more distinct amplitudes.

might look different from the principle progression shown in Fig. 14 especially with regard to the maximum occupation of state B. Therefore, the occupation of the degraded state was calculated in respect to the temperature to predict in which temperature range a verification could make sense. As pictured in Fig. 15 the maximum observable occupation of the degraded state B exhibits a noticeable temperature dependence. It is advisable to choose a temperature range in which the occupation of the degraded state becomes large and especially distinguishable from an expectable slight degradation of the sample until the measurement takes place which may occur already at very low light intensities around 1/100 sun. If at least $10 \%$ amplitude is desired, the advisable temperature range would lie below $70{ }^{\circ} \mathrm{C}$. Unfortunately, the destabilization reaction is very slow at those temperatures and takes a hundred days and more. Furthermore, if the occupation of the degraded state is relatively small, single measurements could already affect the experiment. So far, this experiment could not be performed.

\section{CONCLUSIONS}

An analytical model describing the degradation and regeneration process in oxygen-rich boron doped crystalline silicon was introduced and a very good agreement with experimental data could be demonstrated. The reactions between the different states of the 3-state model are described by reaction rates. Their ratio is influenced by the experimental conditions like, e.g., temperature, light intensity, or cur- rent density. As only effective reaction rates can be extracted from experimental data, the presented mathematical description allows for the determination of the individual reaction rates between the three states. Furthermore, the analytical model allows to calculate the occupation of the different states at any time and especially in the long time limit. In combination with experimentally determined reaction rates, some general properties of the model could be verified. An experimental procedure for the clarification of the destabilization path was suggested but not realized yet due to the extremely time-consuming procedure (the model predicts a duration of the temperature treatment of at least 100 days).

${ }^{1}$ J. Schmidt, Prog. Photovoltaics 13, 325 (2005).

${ }^{2} \mathrm{H}$. Fischer and W. Pschunder, Proceedings of the 10th IEEE Photovoltaic Specialists Conference, Palo Alto, CA, 1973 (IEEE, New York, 1973), p. 404.

${ }^{3}$ V. G. Weizer, H. W. Brandhorst, J. D. Broeder, R. E. Hart, and J. H. Lamneck, J. Appl. Phys. 50, 4443 (1979).

${ }^{4}$ J. Schmidt, A. G. Aberle, and R. Hezel, Proceedings of the 26th IEEE Photovoltaic Specialists Conference, Anaheim, CA, 1997 (IEEE, New York, 1997), p. 13.

${ }^{5}$ S. W. Glunz, E. Schäffer, S. Rein, K. Bothe, and J. Schmidt, Proceedings of the 3rd World Conference on Photovoltaic Energy Conversion, Osaka, Japan (Arisumi, Japan, 2003), p. 919.

${ }^{6}$ K. Bothe and J. Schmidt, J. Appl. Phys. 99, 013701 (2006).

${ }^{7}$ K. Bothe, R. Sinton, and J. Schmidt, Prog. Photovoltaics 13, 287 (2005).

${ }^{8}$ J. C. Bourgoin and J. W. Corbett, Phys. Lett. A 38, 135 (1972).

${ }^{9}$ J. Adey, R. Jones, D. W. Palmer, P. R. Briddon, and S. Öberg, Phys. Rev. Lett. 93, 055504 (2004).

${ }^{10}$ A. Herguth, G. Schubert, M. Kaes, and G. Hahn, Proceedings of the 4th World Conference on Photovoltaic Energy Conversion, Hawaii (IEEE, New York, 2006), p. 940.

${ }^{11}$ A. Herguth, G. Schubert, M. Kaes, and G. Hahn, Proceedings of the 21 st European Photovoltaic Solar Energy Conference, Dresden, Germany (WIP Renewable Energies, Munich, 2006), p. 530.

${ }^{12}$ A. Herguth, G. Schubert, M. Kaes, and G. Hahn, Prog. Photovoltaics 16, 135 (2008).

${ }^{13}$ S. Dubois, N. Enjalbert, J. P. Garandet, R. Monna, and J. Kraiem, Proceedings of the 23rd European Photovoltaic Solar Energy Conference, Valencia, Spain (WIP Renewable Energies, Munich, 2008), p. 1437.

${ }^{14}$ B. Lim, S. Hermann, K. Bothe, J. Schmidt, and R. Brendel, Proceedings of the 23rd European Photovoltaic Solar Energy Conference, Valencia, Spain (WIP Renewable Energies, Munich, 2008), p. 1018.

${ }^{15}$ W. Shockley and W. T. Read, Phys. Rev. 87, 835 (1952).

${ }^{16}$ R. A. Sinton and A. Cuevas, Appl. Phys. Lett. 69, 2510 (1996).

${ }^{17}$ Spectrum ASTM G173-03e1, DOI:

${ }^{18}$ J. Schmidt, K. Bothe, and R. Hezel, Proceedings of the 3rd World Conference on Photovoltaic Energy Conversion, Osaka, Japan (Arisumi, Japan, 2003), p. 1077. 